\title{
Possible involvement of pericytes in intraplaque hemorrhage of carotid artery stenosis
}

\author{
*Tatsuya Tanaka, MD, 1,2 Atsushi Ogata, MD, PhD,1 Jun Masuoka, MD, PhD, ${ }^{1}$ \\ Taichiro Mizokami, MD, ${ }^{1,3}$ Tomihiro Wakamiya, MD, PhD, ${ }^{1}$ Yukiko Nakahara, MD, PhD, ${ }^{1}$ \\ Kohei Inoue, MD, PhD, ${ }^{1}$ Shoko Shimokawa, MD, PhD, ${ }^{1}$ Fumitaka Yoshioka, MD, ${ }^{1}$ \\ Nobuaki Momozaki, MD, PhD, ${ }^{2}$ Shuji Sakata, MD, PhD, ${ }^{3}$ and Tatsuya Abe, MD, PhD ${ }^{1}$
}

${ }^{1}$ Department of Neurosurgery, Faculty of Medicine, Saga University, Saga; '2Department of Neurosurgery, Imari Arita Kyoritsu Hospital, Imari; and ${ }^{3}$ Department of Neurosurgery, Saga Ken Medical Centre Koseikan, Saga, Japan

OBJECTIVE Intraplaque hemorrhage (IPH) is most often caused by the rupture of neovessels; however, the factors of intraplaque neovessel vulnerability remain unclear. In this study, the authors focused on pericytes and aimed to investigate the relationship between IPH and pericytes.

METHODS The authors retrospectively analyzed the medical records of all patients with carotid artery stenoses who had undergone carotid endarterectomy at their hospitals between August 2008 and March 2016. Patients with carotid plaques that could be evaluated histopathologically were eligible for study inclusion. Intraplaque hemorrhage was analyzed using glycophorin A staining, and patients were divided into the following 2 groups based on the extent of granular staining: high IPH (positive staining area $>10 \%$ ) and low IPH (positive staining area $\leq 10 \%$ ). In addition, intraplaque neovessels were immunohistochemically evaluated using antibodies to CD34 as an endothelial cell marker or antibodies to NG2 and CD146 as pericyte markers. The relationship between IPH and pathology for intraplaque neovessels was investigated.

RESULTS Seventy of 126 consecutive carotid stenoses were excluded due to the lack of a specimen for histopathological evaluation; therefore, 53 patients with 56 carotid artery stenoses were eligible for study inclusion. Among the 56 stenoses, 37 lesions had high IPH and 19 had low IPH. The number of CD34-positive neovessels was equivalent between the two groups. However, the densities of NG2- and CD146-positive neovessels were significantly lower in the high IPH group than in the low IPH group (5.7 \pm 0.5 vs. $17.1 \pm 2.4, p<0.0001 ; 6.6 \pm 0.8$ vs. $18.4 \pm 2.5, p<0.0001$, respectively).

CONCLUSIONS Plaques with high IPH are associated with fewer pericytes in the intraplaque neovessels. This finding may help in the development of novel therapeutic strategies targeting pericytes.

https://thejns.org/doi/abs/10.3171/2018.1.JNS171942

KEYWORDS carotid artery; carotid stenosis; pericyte; vascular disorders

$\mathrm{C}$ AROTID artery stenosis is a risk factor for cerebral infarction, and when associated with an unstable plaque, it can progress to atheroembolism..$^{15,16} \mathrm{Un}$ stable plaques are characterized either by a thin fibrous cap with a lipid-rich necrotic core or by intraplaque hemorrhage (IPH). ${ }^{9}$ In particular, plaque neovascularization, IPH, and plaque rupture are highly related to adverse events. ${ }^{12,17,25,26} \mathrm{~A}$ recent study showed that the neovessel density was not significantly different between a condition with and that without IPH. ${ }^{27}$ We hypothesized that IPH is associated with the vulnerability of intraplaque neovessels and focused on pericytes. Pericytes encircle endothelial cells in capillary vessels in almost all tissues and are important for maturation and stabilization of the capillary during angiogenesis. ${ }^{2}$ The proportion of pericytes to endothelial cells, i.e., pericyte coverage, is markedly higher in the brain than in other organs, indicating the importance of pericytes in the brain. ${ }^{2}$ The brain pericytes are reported to contribute to the formation and maintenance of the blood-brain barrier, through which transport of molecules

ABBREVIATIONS CEA = carotid endarterectomy; IPH = intraplaque hemorrhage.

SUBMITTED August 7, 2017. ACCEPTED January 17, 2018.

INCLUDE WHEN CITING Published online June 29, 2018; DOI: 10.3171/2018.1.JNS171942.

* T.T. and A.O. share first authorship. 
from the blood to neurons is strictly regulated and brain homeostasis is maintained. ${ }^{3}$ However, the role of pericytes in IPH is unclear. In the present study, we aimed to investigate the relationship between IPH and pericytes.

\section{Methods \\ Patient Population}

This study was approved by the regional ethics committee of Saga University Hospital. We retrospectively analyzed the medical records of all patients with carotid artery stenoses who had undergone carotid endarterectomy (CEA) at Saga University Hospital and affiliated hospitals between August 2008 and March 2016. Patients eligible for inclusion in the study were those with carotid plaques that could be evaluated histopathologically. Carotid endarterectomy was indicated for symptomatic and asymptomatic patients with carotid artery stenosis $>50 \%$ and $>$ $80 \%$, respectively, using the North American Symptomatic Carotid Endarterectomy Trial criteria. A carotid stenosis was considered symptomatic if there had been a previous ischemic event such as stroke, transient ischemic attack, or amaurosis fugax in the 6 months before surgery; it was considered asymptomatic if there was no such history. We also recorded the incidence of cardiovascular risk factors.

\section{Immunohistochemical Analysis}

Carotid plaques obtained during CEA were fixed in formalin immediately after surgical removal and divided transversely at the maximum point of the macroscopic stenotic lesion. Approximately 3-mm-thick tissue blocks were fixed in $10 \%$ buffered formalin and embedded in paraffin. Serial $4-\mu \mathrm{m}$ transverse sections were then stained with $\mathrm{H} \& \mathrm{E}$, and the adjacent sections were used for immunohistochemical analysis. Immunohistochemical staining was performed for glycophorin A (dilution 1:800, DAKO), CD68 (dilution 1:100, DAKO), CD34 (dilution 1:2000, Abcam), NG2 (dilution 1:400, Merck Millipore), and CD146 (dilution 1:800, Abcam).

Intraplaque neovascularization was evaluated using antibodies to CD34, as endothelial cell markers, or antibodies to NG2 and CD146, which have been reported as pericyte markers. ${ }^{5,8}$ In this analysis, a "plaque neovessel" was defined as a lumen that had a single layer and showed immunohistochemical-positive tube-like formation. "Shoulder lesions" were defined as tissues adjacent to the outer border of the lipid core down to the vessel lumen. ${ }^{19}$ Most of the newly formed vessels in atherosclerotic plaques have been reported to develop in shoulder lesions; ${ }^{10}$ therefore, we evaluated the number of neovessels in these areas using two sections of the thickest plaque. These sections were imaged using a microscope-mounted high-resolution digital camera (Olympus). Two certified neurosurgeons who were blinded to the clinical data counted the neovessels using ImageJ software (http://rsb.info.nih.gov/ij/down $\mathrm{load} /$ ), and the mean values were analyzed.

We analyzed for the presence of macrophage infiltration in the shoulder lesions using CD68. The proportion of the CD68-positive area was measured by a certified neurosurgeon (A.O.) using ImageJ software. Next, we analyzed IPH using glycophorin A as a protein specific to the erythrocyte membrane. ${ }^{11}$ Glycophorin A grades have been described as follows in coronary arteries: 1 , focal granular staining in less than $5 \%$ of the plaque; 2 , mild granular staining in $5 \%-10 \%$ of the plaque; 3 , moderate granular staining in $11 \%-25 \%$ of the plaque; and 4, marked granular staining in more than $25 \%$ of the plaque. ${ }^{11}$ Using these grades, we divided the patients into the following 2 groups: low IPH group (grades 1 and 2) and high IPH group (grades 3 and 4). A certified neurosurgeon (A.O.) measured the proportion of the glycophorin A-positive area using ImageJ software.

Finally, neovessel caliber was assessed by a certified neurosurgeon (A.O.). The shortest diameter was defined as the neovessel caliber. We compared the caliber of NG2and CD146-positive neovessels with that of NG2- and CD146-negative neovessels.

\section{Statistical Analysis}

Nominal data were analyzed using Fisher's exact test. Parametric and nonparametric numerical data were analyzed using the t-test and Mann-Whitney U-test, respectively. Correlations were examined using Spearman's rank correlation test. Interobserver variability in the number of neovessels was examined using a paired t-test. We used JMP Pro 11 software (SAS Institute Inc.) for the statistical analyses. A p value $<0.05$ was considered statistically significant.

\section{Results}

Seventy of 126 consecutive carotid stenoses were excluded given the lack of a specimen for histopathological evaluation. Thus, 53 patients with 56 carotid artery stenoses were eligible for analysis (Fig. 1). There were 50 men and 3 women, and the mean patient age was 70 years (range 38-87 years). Among the 56 lesions, 43 (77\%) were symptomatic and $13(23 \%)$ were asymptomatic. The patients had a high incidence of cardiovascular risk factors, including hypertension (41 lesions [73\%]) and dyslipidemia (29 lesions [52\%]; Table 1).

On glycophorin A staining, 37 lesions had high IPH and

TABLE 1. Summary of characteristics among 53 patients with 56 carotid artery stenoses

\begin{tabular}{lccc}
\hline \multicolumn{1}{c}{ Variable } & High IPH & Low IPH & p Value \\
\hline No. of lesions & 37 & 19 & \\
\hline Age in yrs & $72.5 \pm 7.5$ & $66.4 \pm 13.8$ & 0.035 \\
\hline Male sex & $35(95)$ & $18(95)$ & NS \\
\hline Rate of stenosis & $70.5 \pm 14.5$ & $80.4 \pm 14.9$ & 0.02 \\
\hline Symptomatic lesions & $29(78)$ & $14(74)$ & NS \\
\hline Hypertension & $25(68)$ & $16(84)$ & NS \\
\hline Dyslipidemia & $21(57)$ & $8(42)$ & NS \\
\hline Diabetes mellitus & $17(46)$ & $9(47)$ & NS \\
\hline Coronary artery disease & $15(41)$ & $6(32)$ & NS \\
\hline Smoking & $20(54)$ & $13(68)$ & NS \\
\hline
\end{tabular}

NS = not significant.

Data are presented as the mean \pm standard deviation or number (\%), unless indicated otherwise. $p<0.05$ considered statistically significant. 


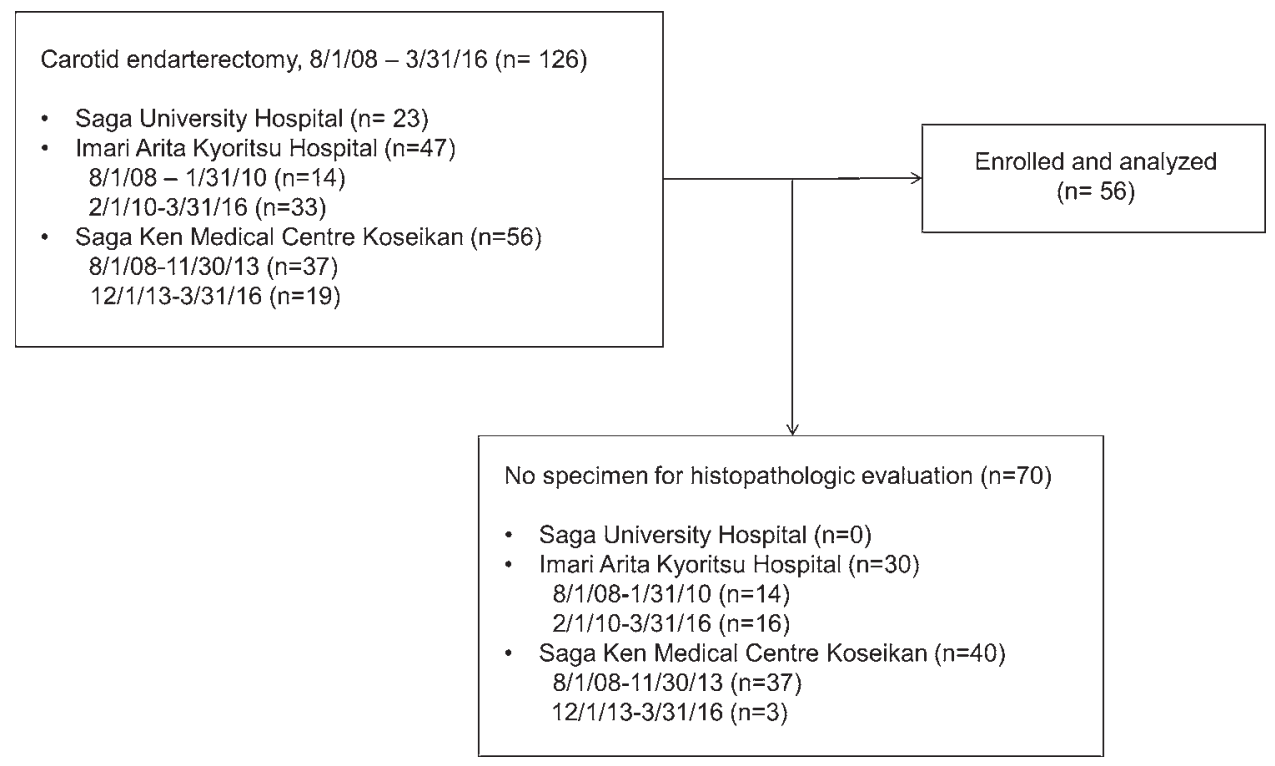

FIG. 1. Flow diagram of patients enrolled and analyzed.

19 had low IPH, and these cases were classified into the high and low IPH groups, respectively. The mean number of CD34-positive neovessels was similar in the high and low IPH groups $(10.3 \pm 0.8$ vs. $10.8 \pm 1.4, p=0.75$; Fig. $2 \mathrm{~A})$. The mean number of NG2-positive neovessels was significantly lower in the high IPH group than in the low IPH group $(5.7 \pm 0.5$ vs. $17.1 \pm 2.4, p<0.0001$; Fig. $2 \mathrm{~B})$. Additionally, the mean number of CD146-positive neoves- sels was significantly lower in the high IPH group than in the low IPH group ( $6.6 \pm 0.8$ vs. $18.4 \pm 2.5$, p $<0.0001$; Fig. $2 \mathrm{C})$. Similar results were obtained when two observers analyzed the results separately; interobserver variability was low (Table 2). The mean number of NG2- and CD146positive neovessels was significantly correlated (Fig. 2D).

The CD68-positive area was significantly larger in lesions with high IPH than in those with low IPH (Fig.
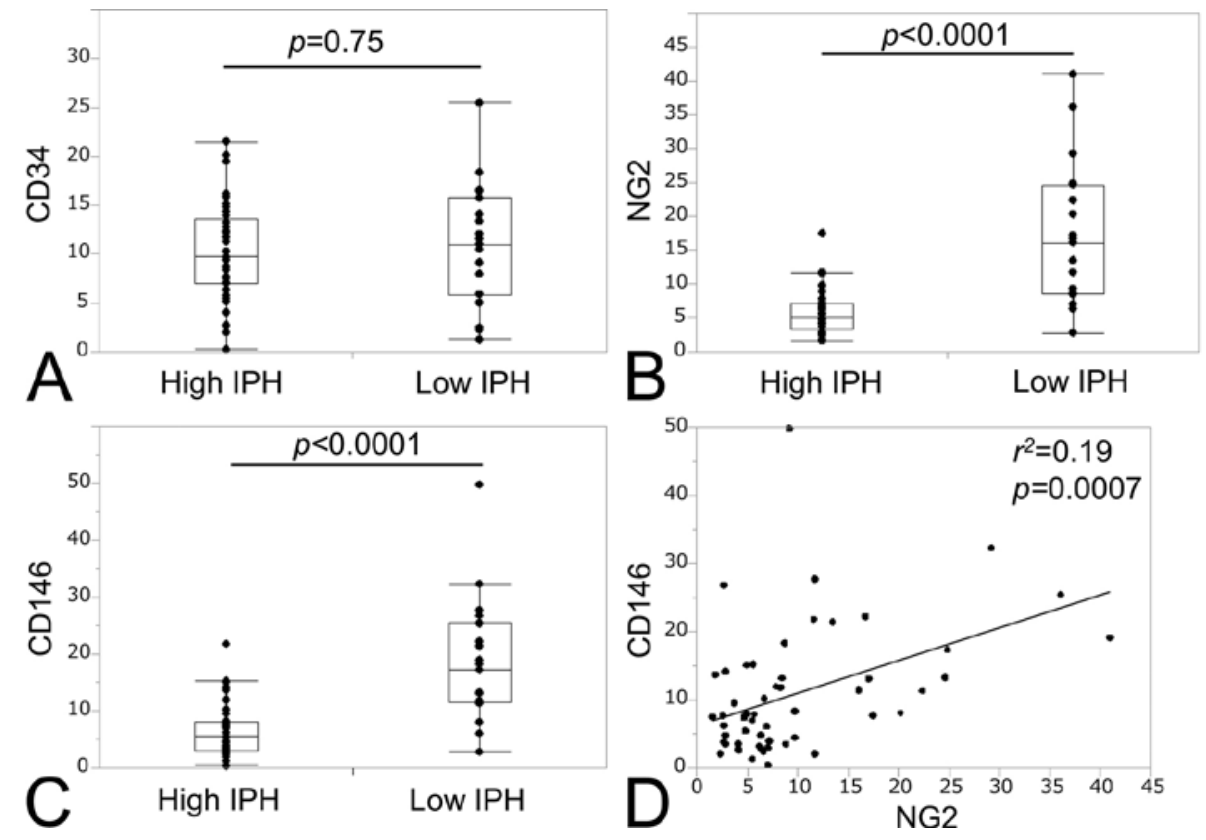

FIG. 2. Box plots showing the relationship between the number of neovessels and IPH. No significant difference was found in the number of CD34-positive neovessels between the high and low IPH groups (A). The number of NG2-positive neovessels was significantly lower in the high IPH group than in the low IPH group (B). The number of CD146-positive neovessels was significantly lower in the high IPH group than in the low IPH group (C). Horizontal lines within the bars represent medians. The number of NG2and CD146-positive neovessels was significantly correlated (D). 

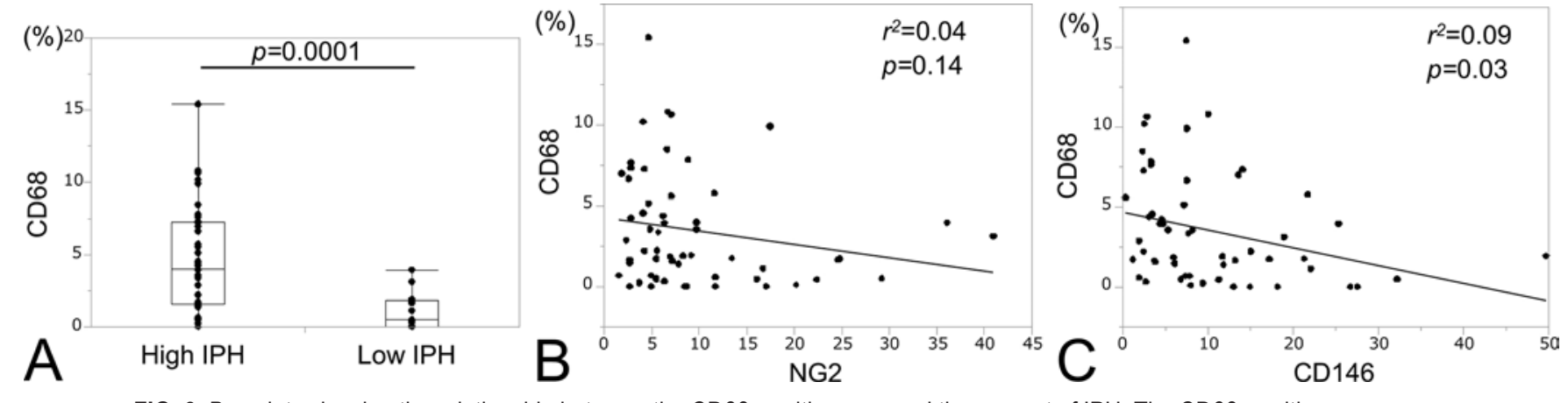

FIG. 3. Box plots showing the relationship between the CD68-positive area and the amount of IPH. The CD68-positive area was significantly larger in lesions with high IPH than in those with low IPH (A). The correlation between the number of NG2-positive neovessels and the CD68-positive area was not significant (B). The correlation between the number of CD146-positive neovessels and the CD68-positive area was slightly significant (C).

3A). The correlation between the mean number of NG2positive neovessels and the CD68-positive area was not significant (Fig. 3B). The correlation between the mean number of CD146-positive neovessels and the CD68-positive area was slightly significant (Fig. 3C). The number of neovessels with NG2- and CD146-positive staining was significantly higher and the CD68-positive area was sig- nificantly smaller in the low IPH group than in the high IPH group (Fig. 4).

The NG2- and CD146-negative stained neovessels were frequently enlarged compared with the neovessels that stained positive with NG2 $(8.6 \pm 0.6 \mu \mathrm{m}$ vs. $25.7 \pm 2.1$ $\mu \mathrm{m}, \mathrm{p}<0.0001)$ and CD146 (9.0 $\pm 0.4 \mu \mathrm{m}$ vs. $31.2 \pm 1.4$ $\mu \mathrm{m}, \mathrm{p}<0.0001$; Fig. 5).
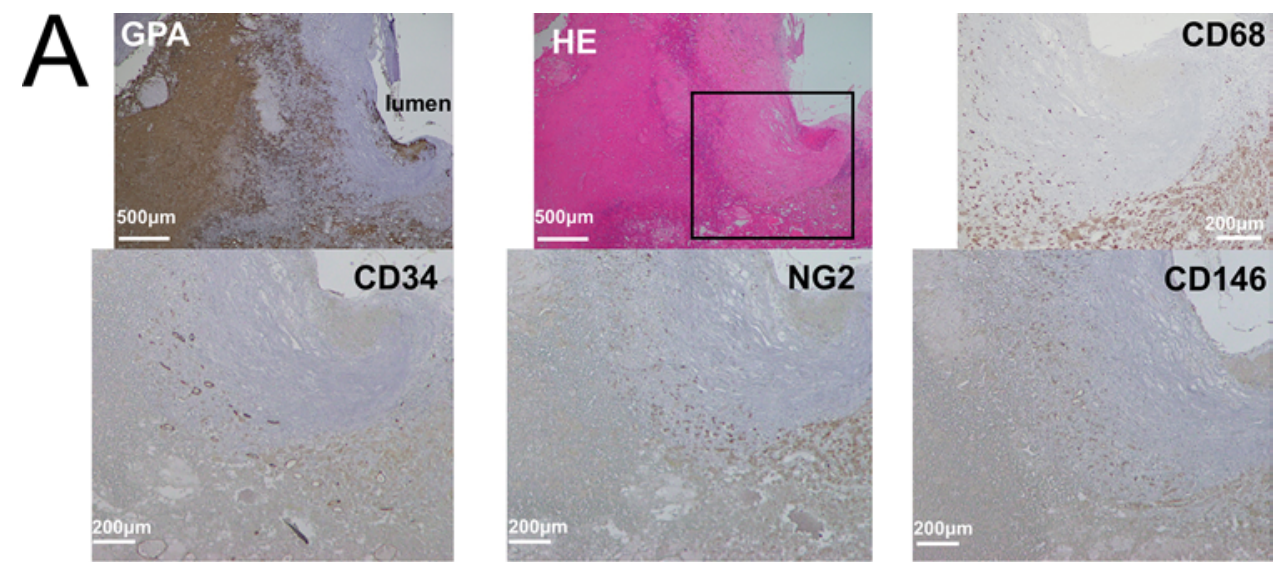

$\mathrm{B}$
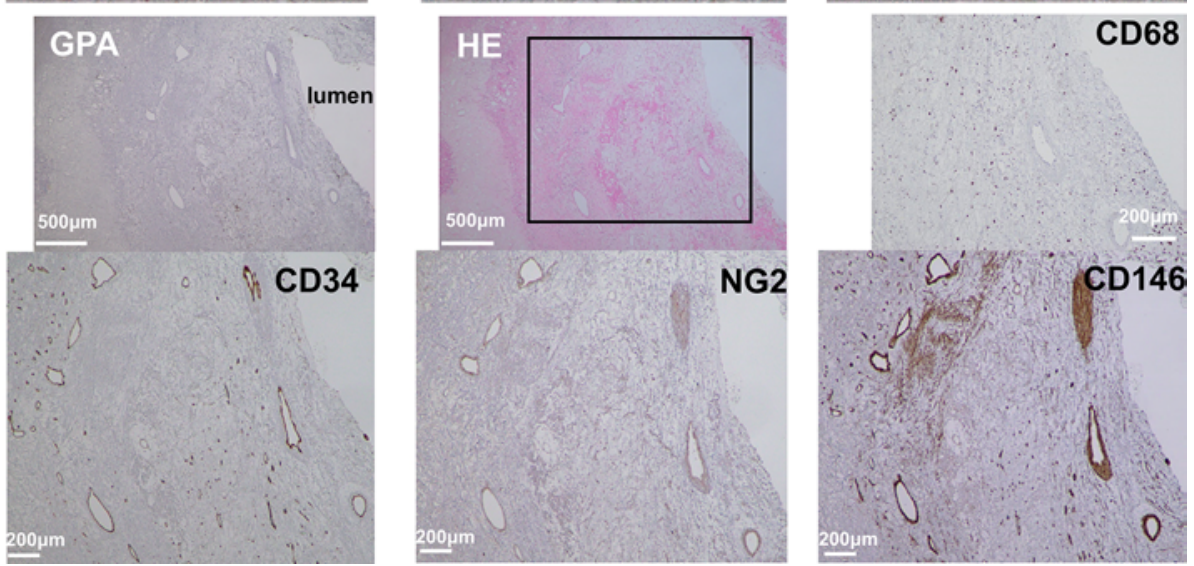

FIG. 4. Representative cases in the high IPH group (A) and the low IPH group (B). Photomicrographs of glycophorin A and CD68 staining show many positive cells in the high IPH group. The number of CD34-positive neovessels was similar in the two groups. The neovessel densities with NG2- and CD146-positive staining were significantly higher in the low IPH group than in the high IPH group. Square outlines in the panels stained using H \& E show plaque shoulder lesions. Original magnification $\times 40 . G P A=$ glycophorin A. Figure is available in color online only. 
TABLE 2. Interobserver variability in measuring the number of neovessels

\begin{tabular}{|c|c|c|c|c|}
\hline \multirow[b]{2}{*}{ Variable } & \multicolumn{3}{|c|}{ Mean No. of Neovessels } & \multirow{2}{*}{$\begin{array}{l}\mathrm{p} \text { Value for } \\
\text { Interobserve } \\
\text { Variability* }\end{array}$} \\
\hline & High IPH & Low IPH & p Value & \\
\hline \multicolumn{5}{|l|}{ CD34 } \\
\hline Observer 1 & $10.4 \pm 1.1$ & $12.0 \pm 1.6$ & 0.39 & 0.39 \\
\hline Observer 2 & $10.2 \pm 1.1$ & $9.6 \pm 1.9$ & 0.74 & \\
\hline \multicolumn{5}{|l|}{ NG2 } \\
\hline Observer 1 & $5.1 \pm 0.5$ & $21.4 \pm 3.0$ & $<0.0001$ & 0.047 \\
\hline Observer 2 & $6.5 \pm 0.7$ & $12.8 \pm 2.0$ & 0.007 & \\
\hline \multicolumn{5}{|l|}{ CD146 } \\
\hline Observer 1 & $5.8 \pm 0.9$ & $18.4 \pm 3.9$ & 0.0001 & 0.53 \\
\hline Observer 2 & $7.4 \pm 1.1$ & $18.4 \pm 2.8$ & $<0.0001$ & \\
\hline
\end{tabular}

*Paired t-test.

The area with positive glycophorin A staining tended to be greater in symptomatic than in asymptomatic patients $(22.7 \% \pm 2.7 \%$ vs. $14.4 \% \pm 3.0 \%, \mathrm{p}=0.12)$. No differences were found between symptomatic and asymptomatic patients in terms of the CD68-positive area (3.6 \pm $0.6 \%$ vs. $3.0 \pm 0.8 \%, p=0.54$ ) and the mean number of CD34-positive vessels $(10.5 \pm 0.9$ vs. $10.4 \pm 1.2, p=0.98)$, NG2-positive vessels $(9.7 \pm 1.4$ vs. $9.1 \pm 2.0, p=0.79)$, and CD146-positive vessels $(10.6 \pm 1.2$ vs. $10.7 \pm 3.5, \mathrm{p}=0.95)$.

\section{Discussion}

In this study, we showed higher expressions of NG2 and
CD146 in the intraplaque neovessels with low IPH than in those with high IPH and no significant difference in the expression of CD34 between low and high IPH groups. The causative factor of IPH may not be fewer endothelial cells but may instead involve pericytes in the intraplaque neovessels. Arterial pericytes in advanced and complicated atherosclerotic lesions may be involved in vascular remodeling, calcification, and intraplaque neovascularization. ${ }^{21}$ The role of pericytes in carotid plaques has been mentioned in only one report, which showed that pericytes were associated with asymptomatic carotid stenosis and calcified plaque. $^{5}$ Additionally, the authors showed that human vascular pericytes secreted osteoprotegerin, which underwent osteoblastic differentiation in vitro, and they suggested that pericytes may contribute to the stabilization of carotid plaques. ${ }^{5}$ The present study showed the possible involvement of pericytes in IPH of carotid stenosis.

Recently, several authors have reported on the role of pericytes in experimental ischemic stroke models. Studies have reported an enlargement in the infarct volume due to insufficient blood-brain barrier formation with increasing vascular permeability after ischemic stroke in pericytedeficient mice compared with that in wild-type mice. ${ }^{4,18,23}$ Pericyte loss in diabetic retinopathy can advance endothelial cell loss, resulting in the formation of microaneurysms, which cause retinal hemorrhage. ${ }^{7,8}$ In the present study, we showed the enlargement of intraplaque neovessels without the expression of pericytes compared to that with the expression of pericytes. Therefore, pericyte loss in intraplaque neovessels may cause IPH.

We showed the possible involvement of pericytes in IPH of carotid stenosis. The relationships between
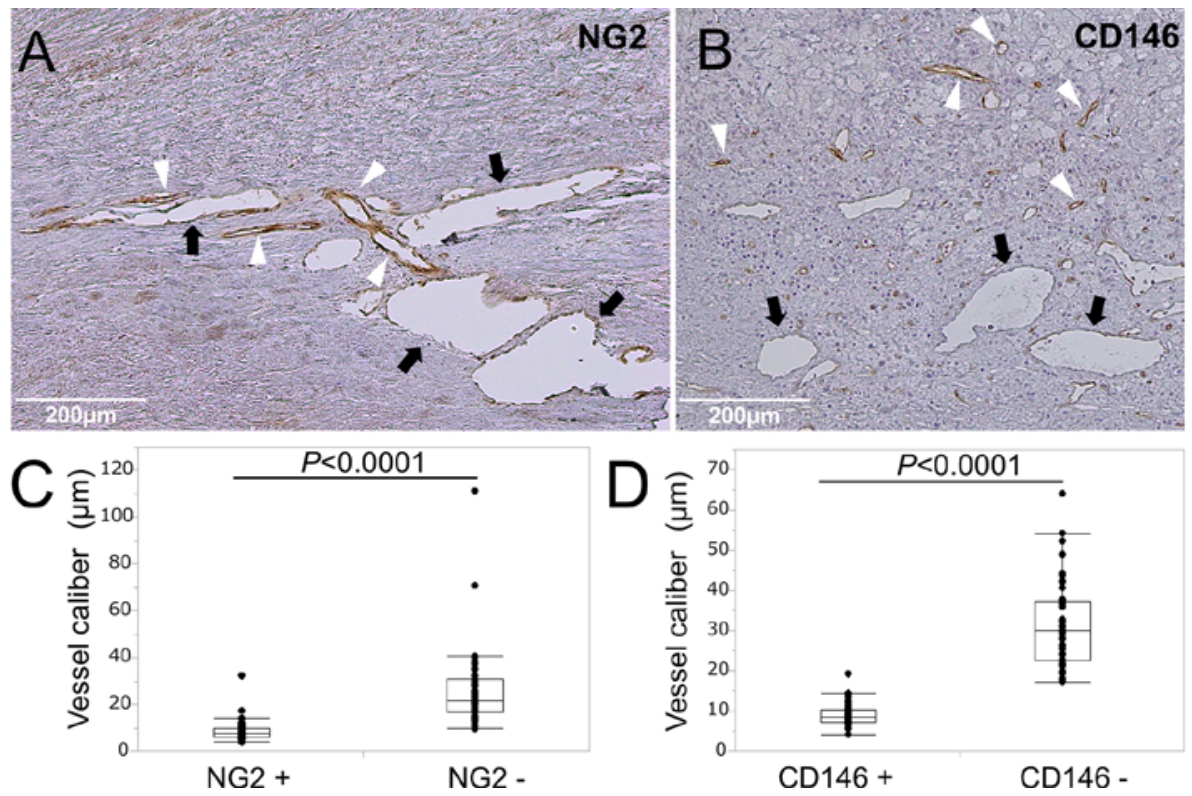

FIG. 5. Photomicrographs of NG2 (A) and CD146 (B) staining show enlargement of negatively stained neovessels (black arrows) compared with the size of positively stained neovessels (white arrowheads). Box plot (C) showing the relationship of vessel caliber with the results of NG2 staining. The caliber of NG2-negative vessels was significantly larger than that of NG2-positive vessels. Box plot (D) showing the relationship of vessel caliber with the results of CD146 staining. The caliber of CD146-negative vessels was significantly larger than that of CD146-positive vessels. Figure is available in color online only. 
pericytes and drugs have been described by several authors. ${ }^{20,24}$ In an experimental ischemic stroke animal study, only cilostazol, as compared to other antiplatelet drugs such as aspirin and clopidogrel, promoted angiogenesis with the proliferation of pericytes in the peri-infarct area. ${ }^{20}$ Takagi et al. showed that cilostazol induced the proliferation of pericytes in vitro and contributed to the reduction in cerebral hematoma volume in experimental cerebral hemorrhage models. ${ }^{24}$ Therefore, it is conceivable that the use of cilostazol may enhance pericyte function and reduce the occurrence of IPH. Medical therapy for carotid stenosis, particularly in asymptomatic patients, has improved. ${ }^{1}$ Nevertheless, among patients with asymptomatic carotid stenosis, ipsilateral ischemic cerebrovascular events occurred at a higher rate in those with IPH than in those with stable plaques. ${ }^{6}$ In addition, the risk for recurrent infarction is higher in symptomatic carotid stenosis patients with IPH than in those without IPH, despite undergoing current secondary prevention therapy. Liem et al. conducted a prospective study on the association between antiplatelet agents and IPH in carotid stenosis patients. ${ }^{13}$ These authors showed that the previous use of antiplatelet agents was significantly associated with the occurrence of IPH and that aspirin was used in $70 \%$ of the patients who had used antiplatelet agents. ${ }^{13}$ The appropriate medical therapy for carotid stenosis patients with IPH remains unclear. To establish treatments targeting pericytes, it may be possible to tailor treatments according to the plaque characteristics assessed by noninvasive imaging modalities such as MRI.

The present study has a few limitations. First, because of the cross-sectional study design, we can only infer an association between the factors and not a causative mechanism. Second, the sample size is small, and larger patient numbers are required. Third, findings of the present study may have implications for the healing process of IPH since pericytes are multipotent cells and differentiate not only into mural cells but also into fibroblasts. ${ }^{14}$ The present study also showed that the relationship between macrophage infiltration and the expression of pericytes in the intraplaque neovessels was weak. In contrast, one study reported that plaque vulnerability was associated with CD146 expression on macrophages infiltrating human atherosclerotic plaques..$^{22}$ Therefore, the relationship between macrophage infiltration and pericytes of intraplaque neovessels is unclear. Further studies are required to clarify the relationship between pericytes and the vulnerability of plaque neovessels.

\section{Conclusions}

Plaques with high IPH are associated with fewer pericytes in the intraplaque neovessels. This finding may help in the development of novel therapeutic strategies targeting pericytes.

\section{Acknowledgments}

The authors thank Mrs. Yumiko Oishi and Akiko Soejima, Department of Neurosurgery, Faculty of Medicine, Saga University, for their excellent secretarial assistance and Dr. Sho Komukai, Clinical Research Center, Saga University Hospital, for advice on the statistical analysis.
This work was supported by JSPS KAKENHI Grant Numbers 15K10337, 16K10758, and 17K16653.

\section{References}

1. Abbott AL: Medical (nonsurgical) intervention alone is now best for prevention of stroke associated with asymptomatic severe carotid stenosis: results of a systematic review and analysis. Stroke 40:e573-e583, 2009

2. Armulik A, Genové G, Betsholtz C: Pericytes: developmental, physiological, and pathological perspectives, problems, and promises. Dev Cell 21:193-215, 2011

3. Armulik A, Genové G, Mäe M, Nisancioglu MH, Wallgard $\mathrm{E}$, Niaudet C, et al: Pericytes regulate the blood-brain barrier. Nature 468:557-561, 2010

4. Bell RD, Winkler EA, Sagare AP, Singh I, LaRue B, Deane $\mathrm{R}$, et al: Pericytes control key neurovascular functions and neuronal phenotype in the adult brain and during brain aging. Neuron 68:409-427, 2010

5. Davaine JM, Quillard T, Brion R, Lapérine O, Guyomarch $\mathrm{B}$, Merlini T, et al: Osteoprotegerin, pericytes and bone-like vascular calcification are associated with carotid plaque stability. PLoS One 9:e107642, 2014

6. Esposito-Bauer L, Saam T, Ghodrati I, Pelisek J, Heider P, Bauer M, et al: MRI plaque imaging detects carotid plaques with a high risk for future cerebrovascular events in asymptomatic patients. PLoS One 8:e67927, 2013

7. Frank RN: Diabetic retinopathy. N Engl J Med 350:48-58, 2004

8. Hall AP: Review of the pericyte during angiogenesis and its role in cancer and diabetic retinopathy. Toxicol Pathol 34:763-775, 2006

9. Hennerici MG: The unstable plaque. Cerebrovasc Dis 17 (3 Suppl 3): 17-22, 2004

10. Jeziorska M, Woolley DE: Local neovascularization and cellular composition within vulnerable regions of atherosclerotic plaques of human carotid arteries. J Pathol 188:189-196, 1999

11. Kolodgie FD, Gold HK, Burke AP, Fowler DR, Kruth HS, Weber DK, et al: Intraplaque hemorrhage and progression of coronary atheroma. N Engl J Med 349:2316-2325, 2003

12. Kumamoto M, Nakashima Y, Sueishi K: Intimal neovascularization in human coronary atherosclerosis: its origin and pathophysiological significance. Hum Pathol 26:450-456, 1995

13. Liem MI, Schreuder FH, van Dijk AC, de Rotte AA, Truijman MT, Daemen MJ, et al: Use of antiplatelet agents is associated with intraplaque hemorrhage on carotid magnetic resonance imaging: the Plaque at Risk study. Stroke 46:3411-3415, 2015

14. Makihara N, Arimura K, Ago T, Tachibana M, Nishimura A, Nakamura K, et al: Involvement of platelet-derived growth factor receptor $\beta$ in fibrosis through extracellular matrix protein production after ischemic stroke. Exp Neurol 264:127134,2015

15. McCarthy MJ, Loftus IM, Thompson MM, Jones L, London NJ, Bell PR, et al: Angiogenesis and the atherosclerotic carotid plaque: an association between symptomatology and plaque morphology. J Vasc Surg 30:261-268, 1999

16. Mofidi R, Crotty TB, McCarthy P, Sheehan SJ, Mehigan D, Keaveny TV: Association between plaque instability, angiogenesis and symptomatic carotid occlusive disease. Br J Surg 88:945-950, 2001

17. Moreno PR, Purushothaman KR, Sirol M, Levy AP, Fuster $\mathrm{V}$ : Neovascularization in human atherosclerosis. Circulation 113:2245-2252, 2006

18. Nakamura K, Arimura K, Nishimura A, Tachibana M, Yoshikawa Y, Makihara N, et al: Possible involvement of basic FGF in the upregulation of PDGFR $\beta$ in pericytes after ischemic stroke. Brain Res 1630:98-108, 2016 
19. Olson FJ, Strömberg S, Hjelmgren O, Kjelldahl J, Fagerberg B, Bergström GM: Increased vascularization of shoulder regions of carotid atherosclerotic plaques from patients with diabetes. J Vasc Surg 54:1324-1331, 1131.e1-1131.e5, 2011

20. Omote Y, Deguchi K, Kono S, Liu N, Liu W, Kurata T, et al: Neurovascular protection of cilostazol in stroke-prone spontaneous hypertensive rats associated with angiogenesis and pericyte proliferation. J Neurosci Res 92:369-374, 2014

21. Orekhov AN, Bobryshev YV, Chistiakov DA: The complexity of cell composition of the intima of large arteries: focus on pericyte-like cells. Cardiovasc Res 103:438-451, 2014

22. Qian YN, Luo YT, Duan HX, Feng LQ, Bi Q, Wang YJ, et al: Adhesion molecule CD146 and its soluble form correlate well with carotid atherosclerosis and plaque instability. CNS Neurosci Ther 20:438-445, 2014

23. Shen J, Ishii Y, Xu G, Dang TC, Hamashima T, Matsushima T, et al: PDGFR- $\beta$ as a positive regulator of tissue repair in a mouse model of focal cerebral ischemia. J Cereb Blood Flow Metab 32:353-367, 2012

24. Takagi T, Imai T, Mishiro K, Ishisaka M, Tsujimoto M, Ito $\mathrm{H}$, et al: Cilostazol ameliorates collagenase-induced cerebral hemorrhage by protecting the blood-brain barrier. J Cereb Blood Flow Metab 37:123-139, 2017

25. Truijman MT, de Rotte AA, Aaslid R, van Dijk AC, Steinbuch J, Liem MI, et al: Intraplaque hemorrhage, fibrous cap status, and microembolic signals in symptomatic patients with mild to moderate carotid artery stenosis: the Plaque at RISK study. Stroke 45:3423-3426, 2014

26. van den Oord SC, Akkus Z, Renaud G, Bosch JG, van der Steen AF, Sijbrands EJ, et al: Assessment of carotid atherosclerosis, intraplaque neovascularization, and plaque ulceration using quantitative contrast-enhanced ultrasound in asymptomatic patients with diabetes mellitus. Eur Heart J Cardiovasc Imaging 15:1213-1218, 2014

27. Vrijenhoek JE, Den Ruijter HM, De Borst GJ, de Kleijn DP, De Vries JP, Bots ML, et al: Sex is associated with the presence of atherosclerotic plaque hemorrhage and modifies the relation between plaque hemorrhage and cardiovascular outcome. Stroke 44:3318-3323, 2013

\section{Disclosures}

The authors report no conflict of interest concerning the materials or methods used in this study or the findings specified in this paper.

\section{Author Contributions}

Conception and design: Ogata, Wakamiya. Acquisition of data: Ogata, Tanaka, Mizokami, Wakamiya, Nakahara, Inoue, Shimokawa, Yoshioka, Momozaki. Analysis and interpretation of data: Ogata, Tanaka. Drafting the article: Ogata. Critically revising the article: Ogata, Masuoka. Reviewed submitted version of manuscript: all authors. Approved the final version of the manuscript on behalf of all authors: Ogata. Statistical analysis: Ogata. Administrative/technical/material support: Tanaka, Masuoka, Mizokami, Nakahara, Inoue, Shimokawa, Yoshioka, Momozaki, Sakata. Study supervision: Ogata, Masuoka, Momozaki, Sakata, Abe.

\section{Correspondence}

Atsushi Ogata: Saga University, Saga, Japan. ogata.a24@gmail. com. 